\title{
Characterizing the mathematical problem-solving strategies of transitioning novice physics students
}

\author{
Eric Burkholder(1) \\ Department of Physics, Stanford University, 382 Via Pueblo, Stanford, California 94305, USA \\ Lena Blackmon \\ Department of Applied Physics, Stanford University, 348 Via Pueblo, Stanford, California 94305, USA \\ Carl Wieman \\ Department of Physics and Graduate School of Education, Stanford University, \\ 382 Via Pueblo, Stanford, California 94305, USA
}

(Received 20 August 2020; accepted 19 October 2020; published 11 November 2020)

\begin{abstract}
Much work has been done to characterize the reasoning of students as they solve mathematics-intensive problems and characterizing differences in expert and novice problem solving. In this work, we characterize the problem-solving strategies in a classroom setting of "transitioning novices," students who have completed an introductory physics course and have learned some problem-solving strategies, but are far from expertlike in their reasoning. We find that students mostly use intermediate strategies that reflect an understanding of specific relationships between quantities, such as analyzing the units of an expression, to reason about mathematical expressions. Few students use more sophisticated strategies like checking limits, which require students to run mental simulations to predict how a system will behave as different physical variables are changed. The teaching of more advanced strategies like limit checking will require careful scaffolding of the cognitive complexity, as students generally do not succeed when simply told to check limits. This is supported by the findings of Lin and Singh [Phys. Rev. Phys. Educ. Res. 7, 020104 (2011)] that careful scaffolding is needed to help students solve more complex problems. In this particular group, students were able to successfully analyze the dimensions of an expression and compute component forces and torques to check if their answer made sense. Our results show that there is a need to recognize and teach these intermediary strategies to enable more novice students to check their answers and encourage students to become more expertlike.
\end{abstract}

DOI: 10.1103/PhysRevPhysEducRes.16.020134

\section{INTRODUCTION}

There has been a substantial amount of work in physics education research dedicated to studying problem solving [1-7], which has shed light on the problem-solving processes of expert physicists. Experts often spend more time planning their approach to problem solving, use more sophisticated mental representations, think about what concepts apply to the problem they are solving, and check whether their answers make sense.

As many physics problems involve mathematical manipulation as well as physical reasoning, researchers have recently begun to focus on how experts and students make physical meaning out of mathematical expressions

Published by the American Physical Society under the terms of the Creative Commons Attribution 4.0 International license. Further distribution of this work must maintain attribution to the author(s) and the published article's title, journal citation, and DOI.
[8-11]. Authors such as Kuo et al. center their works on the idea of cognitive blending: the idea that, to successfully solve physics problems, the student or expert needs to have a mental space that blends elements of mathematical and conceptual physical reasoning $[10,12]$. Brahmia et al. suggest that some essential skills in cognitive blending include "representing concepts symbolically, defining problems quantitatively, and verifying that solutions make sense" [9]. Still, these skills are not exclusive: other authors such as Heller et al. [5], Huffman [13], and Reif [14] articulate similar strategies that involve moving from a conceptual framework toward being able to evaluate a solution for correctness, all as a part of typical physics problems solving.

Other work has specifically investigated students' use of mathematics when solving problems that synthesize multiple different concepts $[1,15]$. These problems require conceptual reasoning to formulate and combine relevant equations, as well as algorithmic manipulation of equations and computation. Ibrahim et al. found that the 
mathematical complexity of a problem (defined by the number and type of equations to be manipulated) had a negative impact on students' problem solving, interfering with not only students' simplification of relevant equations, but also the formulation of equations [15]. Lin and Singh found that students were able to improve their performance on multiconcept problems by learning from worked examples [1]. The authors found that worked examples helped students identify relevant principles (formulate and combine equations), but that more scaffolding was required to help students transfer this knowledge to novel context.

There has also been considerable work looking broadly at expert-novice differences in physics problem solving (see, e.g., Ref. [3]). However, much less is known about when and how students transition from novice to expert problem solvers. To help fill this gap, we conducted an experiment at the end of an introductory mechanics course designed to teach students good problem-solving practices, which we define as students learning to solve problems using strategies identified in the literature on expert problem solving [16]. Students were given one or two symbolic mathematical expressions and asked to reason about the validity of those expressions. In doing this, we had two main research questions.

(1) What types of problem-solving strategies do transitioning novices use in answer checking?

(2) Can the format of the problem affect the way students employ various problem-solving strategies?

\section{BACKGROUND}

There is a large body of work characterizing the differences between experts and novices as they solve physics problems. Differences have been found in expert and novice knowledge organization, qualitative analysis, the depth of disciplinary knowledge, and how they represent the problem. Generally, research has focused on two key differences: differences in procedures and differences in knowledge structures [7].

There are notable differences in how experts and novices structure their knowledge, as well as their relative depth of understanding of relevant physics knowledge. The seminal work in this area is that of Chi et al. [3]. In that work, the researchers showed that novices categorized textbook physics problems according to surface features, such as whether the problems involved an inclined plane, whereas experts categorized these problems according to the physics concepts that were need to solve them. Hardiman et al. showed that this categorization was correlated with more proficient problem solving, but also showed that the experts and novices did not always behave as Chi et al. had described-novices could in some circumstances identify the concepts needed to solve problems and experts could get distracted by surface features [17]. In later work, Chi and co-workers examined differences between "good students" (more expertlike) and "poor students" (more novicelike) as they studied worked examples. They found that the differences in students' self-explanations closely resembled the differences they observed between expert and novice problem categorizations [18]. Dufresne et al. showed the utility of these expert-novice differences for instruction by showing that students could perform expertlike analyses when constrained to following expert protocols [19].

With the exception of Dufresne et al. [19], none of this prior work attempts to examine how problem solvers transition from novice to expert. Here, we build upon this work by exploring the different ways students structure their knowledge in the process of moving from the novice to expert. In line with previous work $[1,15]$, our work suggests that different problem-solving strategies have different cognitive demands, and thus the cognitive complexity needs to be carefully scaffolded in order to allow students to transition from novice to expert.

The participants in this study were 78 (out of 95) students from Physics 41E at Stanford University. Students in this course had little to no high school physics experience (the average force and motion conceptual evaluation prescore was $15 \%$, standard deviation was $7.2 \%$ ) [20]. For the purposes of this study, we call the students who participated in the study "transitioning novices." These students are not complete novices, as they have completed an introductory physics course. In particular, they have completed a course that is specifically designed to teach them to solve problems more like expert physicists. Indeed, in prior research on this course we found that students became better at planning their solutions to problems, reflecting some progress toward expertise [21]. In that same study, however, we found that students were not able to frame the problems like experts (e.g., identify important elements and make assumptions) - further instruction and practice is likely required beyond a single introductory course. Furthermore, as we discuss below, the strategies we observe these students using are markers that they have progressed beyond the phase of being complete novices, and have developed some limited physics expertise.

Physics 41E covers static equilibrium, forces, torques, 1D kinematics, and conservation of energy. This class focuses on teaching students good problem-solving skills through the extensive use of a problem-solving template that explicitly included a step where students were supposed to check their answers [21] (among many other steps). We define good problem solving to mean that students employ similar problem-solving strategies as experts do, as identified by prior research [3]. This means that, for examples, students would learn how to identify the relevant concepts needed to solve a problem without first attempting a solution. The problems students solve in this class are not typical textbook problems, but were all designed by the first and third authors to make sure that 
they involved elements of authentic problem solving [22]. For example, students might be asked to calculate how strong a cable would need to be to support a cantilevered dock. Students would be required to decide what variables their answer might depend on, predict the functional relationship between variables in a problem, make predictions about how the system would behave in limiting cases, and check whether their answer made physical sense. Given the emphasis on practicing these particular expert decisions, we expected, or at least hoped, to see students making these same decisions when we tested them at the end of the course.

\section{METHODS}

The experimental materials consisted of two open-ended questions in two formats each (see Fig. 1). The first question asked how high a person could climb a lightweight ladder leaning against a wall, hereafter called the "ladder problem," and the second question asked what the required strength of an angled rope holding up a shelf and weight was, hereafter called the "shelf problem." Each participant saw one version of each problem.

The problems were presented in two different formats. In the "verify format," students were given a solution to the problem with two mistakes in the final expression-the angular dependence and units of the expression were incorrect. They were directly asked to evaluate whether the dimensions of the expression and the angular

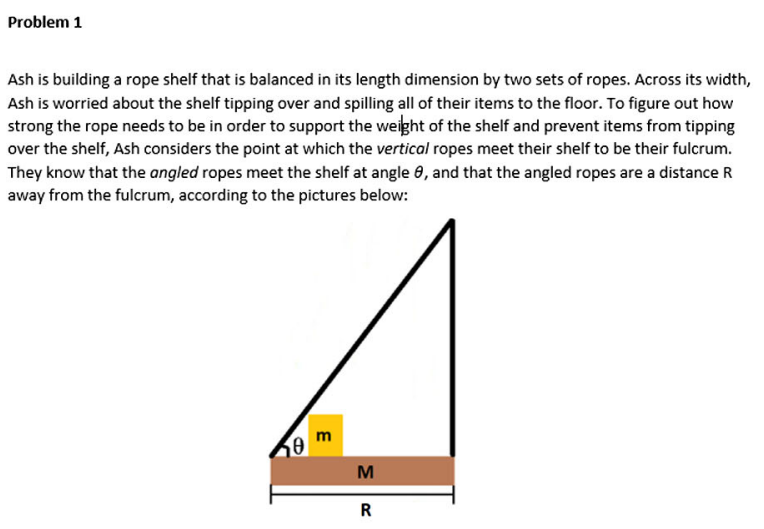

Finally, they plan to place a box of mass $m$ on the shelf at a distance $\frac{2}{3} R$ away from the fulcrum. The shelf itself has a mass of $M$. Ash comes up with the following expression for the required strength of each rope:

$$
F_{\text {rope }}=\frac{7(M+m)}{6 \cos \theta}
$$

1. Does the angle dependence in Ash's solution make sense? Why or why not?

2. Do the units of Ash's solution make sense? Why or why not? dependence were correct. In the "contrasting cases" format of the problem, students were given two solutions: the same incorrect expression as in the verify format as well as the correct solution to the problem. They were asked to evaluate which expression, if either, was correct. We specifically focused our investigation on answer checking because it is well known that experts exhibit this behavior [23,24], but in our experience, it is something that students struggle with. One version of the assessment is shown in Fig. 1.

The experiment was conducted in a two by two format (see Table I). First, all participants read a half-page refresher on static equilibrium and torque. Then participants saw the ladder problem in one of the two formats, and the shelf problem in the second of the two formats The design was balanced to control for the effects of question order. The experiment was an in-class assignment administered through Canvas in the last week of the course. Students were given attendance credit for completing the experiment and were allowed 30 minutes to complete the assignment.

We coded student responses for which strategies students used, and whether that strategy was used successfully. The second author coded all responses, and the first author coded a random $10 \%$ of the responses to establish reliability of the coding scheme (Cohen's $\kappa=0.83$ ). Based on the question prompts, we expected to see students use two strategies.

(1) Evaluating limit (EL): Checking limiting values of the angular dependence of the expressions to check whether the angular dependence is correct.

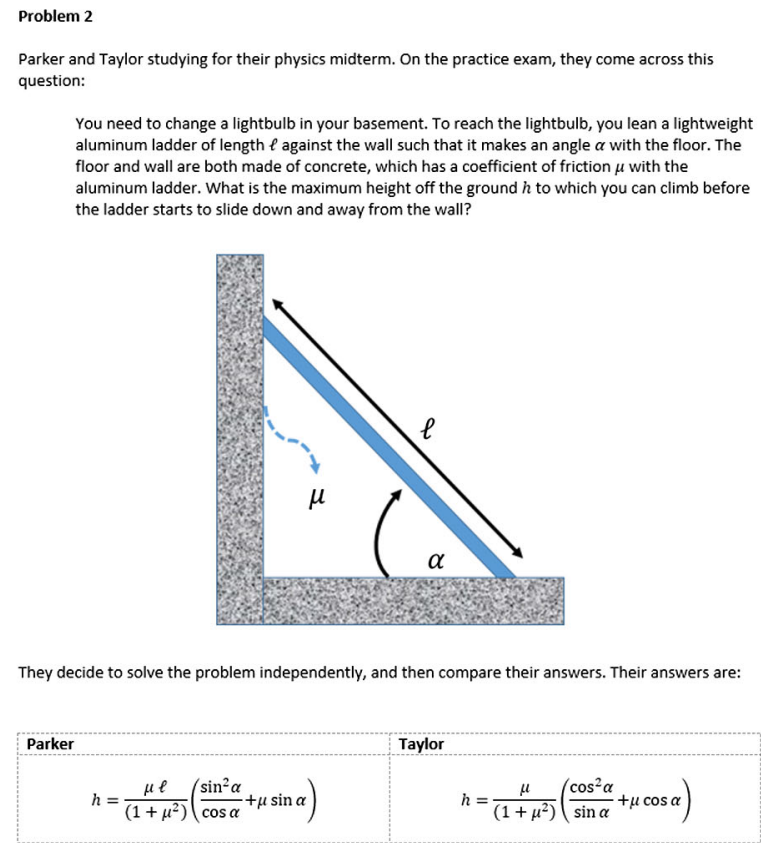

By inspecting these two solutions (without solving), can you determine who has the correct expression: Parker, Taylor, or neither? Please justify your answer.

FIG. 1. Version 3 of the experimental assessment. 
TABLE I. Experimental design. Each group saw one version of the ladder problem and one version of the shelf problem, and the design was balanced to control for the effect of question order.

\begin{tabular}{lcc}
\hline \hline & Contrasting cases first & Verify first \\
\hline Ladder & Group 1 & Group 2 \\
First & $(N=20)$ & $(N=20)$ \\
Shelf & Group 3 & Group 4 \\
First & $(N=21)$ & $(N=17)$ \\
\hline \hline
\end{tabular}

Student example (incorrect): "The angle dependence does make sense because we need to use the angle to find how high we can place the ladder before it starts to slip. This simplifies to $1 / \sin ($ theta) +1 , which does not result in The ladder slipping implies that the angle is getting smaller and smaller (closer to the ground) and therefore, it overcomes the coefficient of friction, resulting in the ladder falling."

(2) Unit analysis (UA): Checking the units of the expression on either side and making sure there is agreement.

Student example (correct): "If we are solving for height $(h)$ we would want an expression that would give us units of height (which they both do). The aspect that is leaning me more towards Parker is that he multiplied the coefficient of friction by the ladder which, in my mind, makes sense because you would have to take the length of the ladder into account."

In addition to these two strategies, we identified a number of other strategies that students used which had been explicitly taught throughout the course.

(1) Identifying components (IC): Deciding on solution correctness based on identified vector components of forces and torques that are relevant to solving the problem.

Student example (incorrect): "The angle dependence in Parker's solution doesn't make sense. You would need sine to determine the height of the ladder however you wouldn't need cosine for that."

(2) Identifying functional relationship (IFR): Identifying the fact that two or more different physical quantities must be mathematically related.

Student example (correct): "W/o solving, I would say that Parker has the correct expression b/c they include all variables (including the length of the ladder), which seems like an important factor to consider in the problem."

(3) Calculating torque (CT): Using the definition $\tau=$ $r F \sin \theta$ to evaluate the solutions given to them.

(4) Do full solution (DFS): Ignoring the explicit instructions to evaluate the solutions without solving the problem themselves and attempting to derive the correct answer.
Each solution was coded for evidence of each strategy, as well as whether the strategy was used successfully. Success in the contrasting cases format was defined by identifying which expression was correct, and success in the verify format was defined by students identifying what the errors were in the expression given to them.

\section{RESULTS}

We find that student problem-solving strategies can be grouped into three categories reflecting the sophistication of students' mental models (see Fig. 2). EL is categorized as a strategy that reflects students' abilities to make predictions. In order to think about limiting cases, students need to have a sophisticated enough mental model of the problem to have certain expectations for the results they will find. This implies that they are able to make predictions about the system to a certain degree and is more reflective of expertise than the other strategies. CT, UA, and IC are all strategies that reflect students' abilities to identify specific mathematical relationships between quantities, e.g., checking the units on both sides of the equation. This requires a certain level of expertise, but is not as expertlike as EL because students do not have to conduct mental simulations using those expressions. IFR is a less sophisticated strategy, reflecting students' ability to identify important factors (e.g., that the height to which you can climb should depend on the coefficient of friction), but generally reflects a failure to identify specific relationships between those factors and is thus less expertlike than CT, UA, and IC. One can see the variations in success rates of various strategies in Fig. 2.

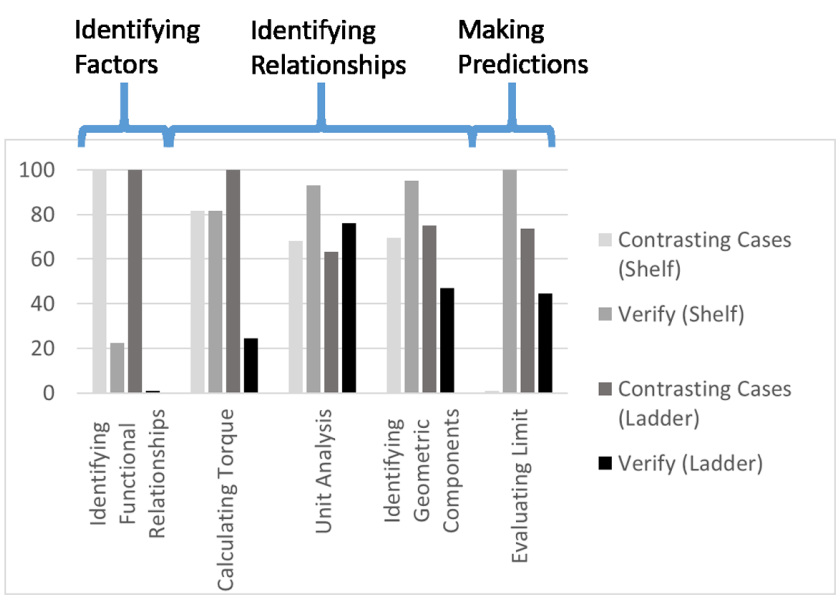

FIG. 2. Fraction of students who used a particular strategy that arrived at the correct answers; $y$ axis measures percentages. This is equivalent to dividing the (correct) column by the total column in Table II. No students used EL in the contrasting cases (shelf) problem, and no student successfully used IFR in the verify (ladder) problem. Strategies are grouped by the level of sophistication. The least sophisticated strategies are identifying factors, next is identifying relationships, and most sophisticated is making predictions. 
TABLE II. Fraction of students who successfully used a strategy to achieve a correct answer and number of total students utilizing a strategy in each format, contrasting cases and verify, for both the shelf and ladder problems. Percentages in each column represent the fraction of total students in each condition.

\begin{tabular}{|c|c|c|c|c|c|c|c|c|}
\hline \multirow[b]{2}{*}{ Strategy } & \multicolumn{4}{|c|}{ Shelf problem } & \multicolumn{4}{|c|}{ Ladder problem } \\
\hline & $\begin{array}{l}\text { Contrasting cases } \\
\text { (Correct) }\end{array}$ & $\begin{array}{l}\text { Contrasting } \\
\text { cases (Total) }\end{array}$ & $\begin{array}{c}\text { Verify } \\
\text { (Correct) }\end{array}$ & $\begin{array}{l}\text { Verify } \\
\text { (Total) }\end{array}$ & $\begin{array}{l}\text { Contrasting cases } \\
\text { (Correct) }\end{array}$ & $\begin{array}{l}\text { Contrasting } \\
\text { cases (Total) }\end{array}$ & $\begin{array}{c}\text { Verify } \\
\text { (Correct) }\end{array}$ & $\begin{array}{l}\text { Verify } \\
\text { (Total) }\end{array}$ \\
\hline Evaluating limit (EL) & $0 \%$ & $0 \%$ & $11 \%$ & $11 \%$ & $8.1 \%$ & $11 \%$ & $12 \%$ & $27 \%$ \\
\hline $\begin{array}{l}\text { Identifying components } \\
\text { (IC) }\end{array}$ & $34 \%$ & $49 \%$ & $59 \%$ & $62 \%$ & $24 \%$ & $32 \%$ & $15 \%$ & $32 \%$ \\
\hline Unit analysis (UA) & $15 \%$ & $22 \%$ & $68 \%$ & $73 \%$ & $24 \%$ & $38 \%$ & $61 \%$ & $80 \%$ \\
\hline Calculating torque (CT) & $22 \%$ & $27 \%$ & $11 \%$ & $13.5 \%$ & $2.7 \%$ & $2.7 \%$ & $2.4 \%$ & $9.8 \%$ \\
\hline $\begin{array}{l}\text { Identifying functional } \\
\text { relationships (IFR) }\end{array}$ & $2.4 \%$ & $2.4 \%$ & $5.4 \%$ & $24 \%$ & $2.7 \%$ & $2.7 \%$ & $0 \%$ & $17 \%$ \\
\hline Do full solution (DFS) & $15 \%$ & $27 \%$ & $2.7 \%$ & $8.1 \%$ & $0 \%$ & $0 \%$ & $0 \%$ & $7.3 \%$ \\
\hline
\end{tabular}

In the figure, we plot the fraction of students who used a particular strategy who arrived at the right answer. The rate of success in Fig. 2 reflects the success rate of individual students, not individual strategies. Students often used multiple strategies to arrive at an answer, so it was not always possible to determine whether their success was the result of one particular strategy.

There was no effect of question order for the students who saw the ladder problem in the contrasting cases format and the shelf problem in the verify format (groups 1 and 4). We counted the number of students who did and did not use a particular strategy in each group, and found no significant difference by Fisher's exact test. For students who saw the ladder problem in the verify format and the shelf problem in the contrasting cases format (groups 2 and 3), we found no difference in strategy use on the ladder problem (using similar methods). Students in group 2 were more likely to use UA (Fisher's exact test, $p=0.007$ ) than students in group 3 when solving the shelf problem, but there were no other differences in strategy use on this problem. Because these differences are minor we conclude the problem order makes little difference. The results in Table I are thus not separated to account for the order in which students saw the questions.

Table II shows that in the shelf problem, IC is a popular strategy in both the contrasting cases and verify format. However, it is used successfully more often in the contrasting cases format than the verify format (95\% success rate in verify format, $69 \%$ success rate in contracting cases format, $p=0.038$ ). UA was far more prevalent in the verify format than the contrasting cases format (used by $73 \%$ of students in verify format, and $22 \%$ of students in contrasting cases format, $p<0.001$ ) and was also used with higher rates of success in the verify format $(93 \%$ success rate in verify format, $68 \%$ success rate in contrasting cases format, $p=0.088)$. CT is used more often in the contrasting cases format than the verify format (27\% versus $13.5 \%$, $p=0.12$ ), but the rate of success with which this strategy is used is the same in both formats $(81 \%$ in both formats, $p=1$ ). IFR and DFS, as tangentially relevant strategies to the task, are rarely successful and used infrequently.

Table II details strategies used in the ladder problem as well. Again, we find that students are more likely to use EL in a verify format than a contrasting cases format $(27 \%$ in verify format, $11 \%$ in contrasting cases format, $p=0.065$ ). Students implement IC at similar levels and with similar rates of success in the contrasting cases and verify formats. Notably, students are much more likely to implement UA in the verify format than the contrasting cases format $(80 \%$ in verify format, $38 \%$ in contrasting cases format, $p<0.001$ ), but they experience similar levels of success in both $(76 \%$ success rate in verify format, $63 \%$ success rate in contrasting cases format, $p=0.49$ ). CT, IFR, and DFS are all used in low numbers and student experience limited success with these strategies.

\section{DISCUSSION}

There are several notable findings in this work. First, we find that students have difficulty readily adopting the expertlike strategy of EL, despite repeated and explicit instruction on this strategy throughout the course. In the contrasting cases formats, between 0 (shelf problem) and $11 \%$ (ladder problem) of students use EL, whereas $11 \%$ and $27 \%$ of students use this strategy when prompted to in the verify format in the shelf and ladder problem, respectively. These difficulties can be understood by considering the level of cognitive complexity required of the mental model. This strategy requires students to have a fairly sophisticated mental model for the problem they are solving, and requires them to be able to predict how changing certain variables will change the behavior of the system.

In both the shelf and ladder problems, students are more likely to use EL when explicitly prompted to (verify format). This is expected for this population because of the explicit instruction they received in using this strategy. This underscores just how unnatural this expert strategy is 
for introductory physics students, and suggests that more work is to be done on how to best teach students to use this strategy. We also note that students who use evaluate limit in a contrasting cases problem are more successful. This suggests that this is indeed a productive strategy for students to learn, if challenging.

UA, which was far more prevalent, requires students to understand relationships between variables (e.g., check that both sides of the expression agree with their prediction of what units are relevant). We found that $24 \%-38 \%$ of students use these strategies in the contrasting cases format and $61 \%-80 \%$ of students use these strategies in the verify format. Most students using this strategy were able to identify that the units of the given expression were incorrect. In addition to this strategy, students relied upon two other strategies that involve identifying relationships between quantities: IC and CT. IFC was more popular than CT (15\%-62\% versus $2.4 \%-27 \%)$. On average, these two strategies were more successful in the shelf problem, because it was a simpler physical system (see below).

Overall, IC and UA were the most commonly used and most successful strategies across problems and problem formats. Both of these strategies reflect students' abilities to identify specific relationships between important quantities in the problem. IC was more common and successful on the shelf problem because the shelf problem is simpler. The relevant components of forces and torques are more easily identified and related to the solution given to the students than they are in the ladder problem. UA was more commonly used on the ladder problem, likely because the dimensional analysis required to identify incorrect units was relatively simple - the quantity of interest was a length instead of a force.

We find that students are more likely to use the expert strategy EL in the ladder problem than the shelf problem. We expect that this is because the ladder problem is more difficult in that the simpler strategies do not work for it. Students cannot identify whether an expression makes physical sense by simply identifying relevant components of forces or which torques need to be calculated. After failing with these simpler strategies, students are driven to use more sophisticated strategies. The ladder problem is a "simultaneous synthesis" problem in that it requires students to simultaneously use multiple different concepts. Ibrahim et al. found that the mathematical complexity of such problems interfered with students' ability to formulate the equations needed to solve the problem, which is consistent with our observation that students struggle to identify relevant equations and thus cannot solve the problem by calculating relevant forces and torques [15]. Identifying components and calculating torques are adequate to reach a solution in the shelf problem, and because they are less cognitively demanding for students, they are more prevalent than in the ladder problem. Given this result and the prevalence of IC in the shelf problem and
UA in the ladder problem, the evidence suggests that students may rely on the simplest productive strategy when checking their answers. This is quite sensible and so, when calling on students to use more difficult strategies like EL, to be convincing, instruction should ensure that such a strategy is needed, and that simpler strategies are inadequate in that context.

The effects of question order were minor because the push to use a particular strategy a student might get from seeing a problem in the verify format first was small compared to the cognitive challenge associated with those strategies. The differences we did see suggest that students were more likely to use an UA if they saw the verify problem first, which is what we expected, but this was different for the ladder and shelf problems and it did not generalize to EL. We expect that it did not generalize to EL because of the wording of the prompt in the verify condition-we prompted students to check the angular dependence and did not explicitly prompt them to check limiting cases. This suggests that students need more explicit guidance to check limiting cases of expressions. It is also interesting that the "shelf-verify" problem was not successful in prompting students to use UA. This may be because students had more difficulty checking the units of force (compared to units of height on the ladder problem).

\section{CONCLUSION}

Our study provides a map of the cognitive developmental trajectory of problem solving and mental models. We found that explicit instruction in expert problem solving encouraged students to adopt useful problem-solving strategies that require them to identify important features of the problem, identify functional relationships between problem features, and predict how the system of interest will behave. Some problem-solving strategies, like EL, are more characteristic of the mental simulations that comprise an expert mental model and expert thinking, while others like IFR represent simpler building blocks of problemsolving reasoning. Notably, we have categorized a suite of problem-solving strategies like UA, IC, and CT that represent an intermediate realm of problem solving in which problem solving is executed via the understanding of mathematical relationships between quantities.

We posit that this intermediary realm is an essential part of transitioning physics learners from novice to expert problem solving, and will likely apply in many areas of physics. Indeed, the use of the intermediate strategies CT, IC, and UA is an indication that students are transitioning from novice to more expert behavior. Thus, instructors should be aware of these strategies not only so that they recognize when their students are transitioning from novice to more expert problem solving, but also so that they can teach students these intermediary strategies as part of scaffolding students on the way to learning more sophisticated answer-checking strategies. These intermediary 
strategies, like UA, have lower cognitive demand than strategies like EL, and will thus be easier for students to learn. Students can employ these strategies productively in relevant contexts while they develop more sophisticated mental models that will allow them to make predictions and check limiting behavior.

Currently, instructors may assume that students acquire the skill of utilizing these strategies without scaffolding; some might assume that students' physical intuition may develop without prompting. Our findings rather suggest that students need explicit guidance to build proficiency in the reasoning strategies that allow them to make sense of their answers. In other words, explicit, deliberate practice in these reasoning strategies, recognizing the differences in the relative cognitive demands, is a necessary component of transitioning students from novice to expert [25]. By analyzing the effect of problem difficulty and formats on the reasoning of transitioning novice students, we are able to characterize these students' reasoning with more precision. The identification of intermediary problem-solving strategies is important for understanding student thinking and how to make instruction more effective.
[1] S.-Y. Lin and C. Singh, Using isomporphic problems to learn introductory physics, Phys. Rev. Phys. Educ. Res. 7, 020104 (2011).

[2] J. Larkin, J. McDermott, D. P. Simon, and H. A. Simon, Expert and novice performance in solving physics problems, Science 208, 1335 (1980).

[3] M. T. H. Chi, P. J. Feltovich, and R. Glaser, Categorization and representation of physics problems by experts and novices, Cogn. Sci. 5, 121 (1981).

[4] J. I. Heller and F. Reif, Prescribing effective human problem-solving processes: Problem description in physics, Cognit. Instr. 1, 177 (1984).

[5] P. Heller, R. Keith, and S. Anderson, Teaching problem solving through cooperative grouping. Part 1: Group versus individual problem solving, Am. J. Phys. 60, 627 (1992).

[6] P. Heller and M. Hollabaugh, Teaching problem solving through cooperative grouping. Part 2: Designing problems and structuring groups, Am. J. Phys. 60, 637 (1992).

[7] L. Hsu, E. Brewe, T. M. Foster, and K. A. Harper, Resource letter RPS-1: Research in problem solving, Am. J. Phys. 72, 1147 (2004).

[8] J. Tuminaro and E. Redish, Elements of a cognitive model of physics problem solving: Epistemic games, Phys. Rev. ST Phys. Educ. Res. 3, 020101 (2007).

[9] S. Brahmia, A. Boudreaux, and S. E. Kanim, Obstacles to mathematization in introductory physics, arXiv $: 1601.01235$.

[10] E. Kuo, M. M. Hull, A. Gupta, and A. Elby, How students blend conceptual and formal mathematical reasoning in solving physics problems, Sci. Educ. 97, 32 (2013).

[11] B. L. Sherin, How students understand physics equations, Cognit. Instr. 19, 479 (2001).

[12] T. J. Bing and E. F. Redish, The cognitive blending of mathematics and physics knowledge, AIP Conf. Proc. 883, 26 (2007).

[13] D. Huffman, Effect of explicit problem solving instruction on high school students problem-solving performance and conceptual understanding of physics, J. Res. Sci. Teach. 34, 551 (1997).

[14] F. Reif, Applying Cognitive Science to Education (MIT Press, Cambridge, MA, 2008).
[15] B. Ibrahim, L. Ding, A. F. Heckler, D. R. White, and R. Badeau, How students process equations in solving quantitative synthesis problems? Role of mathematical complexity in students' mathematical performance, Phys. Rev. Phys. Educ. Res. 13, 020120 (2017).

[16] K. Heller and P. J. Heller, Competent Problem SolverCalculus version (McGraw-Hill, New York, 2000).

[17] P. T. Hardiman, R. Dufresne, and J. P. Mestre, The relationship between problem categorization and problem solving among experts and novices, Mem. Cogn. 17, 627 (1989).

[18] M. T. H. Chi, M. W. Bassok, M. W. Lewis, P. Reimann, and R. Glaser, Self-explanations: How students study and use examples in learning to solve problems, Cogn. Sci. 13, 145 (1989).

[19] R. J. Dufresne, W. J. Gerace, P. T. Hardiman, and J. P. Mestre, Constraining novices to perform expertlike problem analyses: Effects on schema acquisition, J. Learn. Sci. 2, 307 (1992).

[20] R. Thornton and D. Sokoloff, Assessing student learning of Newton's laws: The Force and Motion Conceptual Evaluation and The Evaluation of Active Learning Laboratory and Lecture Curricula, Am. J. Phys. 66, 338 (1998).

[21] E. W. Burkholder, J. K. Miles, K. D. Wang, T. J. Layden, A. V. Fritz, and C. E. Wieman, Template for teaching and assessment of problem-solving in introductory physics, Phys. Rev. Phys. Educ. Res. 16, 010123 (2020).

[22] A. M. Price, C. Kim, E. W. Burkholder, A. V. Fritz, and C. E. Wieman, A universal structure of science and engineering problem-solving, arXiv:2005.11463.

[23] M. P. Čančula, G. Planišič, and E. Etkina, Analyzing patterns in experts' approaches to solving experimental problems, Am. J. Phys. 83, 366 (2015).

[24] C. Singh, When physical intuition fails, Am. J. Phys. 70, 1103 (2002).

[25] K. A. Ericsson, The influence of experience and deliberate practice on the development of superior expert performance, in The Cambridge Handbook of Expertise and Expert Performance, edited by K. A. Ericsson, N. Charness, P. J. Feltovich, and R. R. Hoffman (Cambridge University Press, Cambridge, England, 2006). 